\title{
Improving Safety of a Pilot Plant Reactor using a Model Based Fault Detection and Identification Scheme
}

\author{
Paulo A. F. N. A. Afonso (1), João M. Tavares (2) and José A. A. M. Castro (3) \\ eq2jmc02@eq.uc.pt (1), eq1jmc@eq.uc.pt (3)
}

$(1,3)$ Department of Chemical Engineering, University of Coimbra, Largo Marquês de Pombal, 3000 COIMBRA, PORTUGAL

(2) NARES, Natural Resins Ltd., Santo Varão, 3000 Montemor-o-Velho, PORTUGAL

Keywords: Fault Detection and Identification, Fault Tolerant, Safety, Kalman Filter

\begin{abstract}
This work describes the experimental implementation of an automatic scheme for the on-line detection and identification (FDI) of faults in the sensors of an industrial scale pilot plant reactor under process control, where a pseudo zero-order exothermic chemical reaction is partially simulated. The main goals of this research are to enhance the safety of reactor operations and to demonstrate the potential of FDI for practical industrial applications.

The automatic fault detection and identification method proposed here has two main steps: (1) the detection stage, which relies on a sequential statistical analysis of the process parameters that are continuously estimated by means of a general regression software package (GREG) suitable for non-linear models; (2) the identification step, which is based on an Extended Kalman Filter (EKF) to provide values for the state variables estimates. These values are compared to those given by the sensors thus enabling the identification of the faulty sensor. Moreover, this classification procedure ensures that automatic process control can still be carried on even in such a faulty situation.

Despite the strong non-linearities and the high number of uncertainties, the proposed strategy exhibited very promising results concerning the detection and identification of the faulty sensors. Furthermore, it enabled a satisfactory controller performance for a reasonable period of time, when any of the sensors was disabled and control actions were solely based on state estimates. (C) 1998 Elsevier Science Ltd. All rights reserved.
\end{abstract}

\section{Introduction}

Plants in the chemical and biochemical industries are becoming larger and more complex. The growing environmental and safety demands are now forcing industry to look for new and more powerful techniques for the diagnosis of process faults.

Although important contributions in this subject are reported in the literature, including a few surveys (Isermann, 1984; Gertler, 1988; Frank, 1990), computer simulated plants are often used to illustrate the proposed methodologies. Non-linearities, model mismatch and instrumentation inaccuracies are always present in real chemical processes. They play an important role in FDI algorithms particularly in what concerns false alarms. Therefore, in many cases the robustness of such sophisticated algorithms can not be totally guaranteed when applied to industrial environments.

This paper is concerned with an experimental implementation of an automatic scheme for fault detection and identification in an industrial scale pilot plant, used in a natural resins industry. A stirred tank reactor equipped with a cooling jacket and a hydraulic stirring system composes the core of the plant.

In order to cover a wider range of conditions and also for economy and safety reasons, it is of utmost convenience to partially simulate the chemical reaction (Kershenbaum and Kittisupakorn, 1994). The energy is supplied by introducing steam in the vessel at an appropriate flow rate.

At this stage, our main goal is to detect and identify failures in sensors located in a control loop and to compute the correct control actions. This task is accomplished with a dynamic process model, whose pa- rameters are not well known and time varying. The fault detection method proposed here is based on the process parameters that describe some physical law included in the model. Therefore, a change in some relevant statistical properties of the parameter estimates may indicate that a fault has occurred in the system.

The selected parameters were chosen based on a sensitivity analysis and are continuously estimated by using GREG (Stewart et al., 1992). The fault detection step is performed through a statistical decision test, which analyses the estimates of the process parameters and compares them to the expected properties during normal operation.

In a higher hierarchical level, when a failure condition is detected, the measurements from each sensor are compared with the estimated values obtained through an EKF, enabling the identification of the faulty sensor. In this situation the defective sensor is automatically disabled and process control is carried on through the corresponding state estimate.

\section{Process Description}

The scheme depicted in Fig. 1 represents the pilot plant where the experimental tests were carried out.

The plant is composed by a stirred tank reactor of 80 litre capacity equipped with a cooling jacket. The liquid reactants (water) flow from Tank 1 and Tank 2 with flow rates $Q 1$ and $Q 2$ at temperatures $T 1$ and $T 2$, respectively. The reactor outflow is regulated through control valve VC5, whereas the flow rates $Q 1$ and $Q 2$ are manipulated respectively by control valves $\mathrm{VCl}$ and VC2. The cooling fluid is controlled by means of 
valve VC3, and steam injection into the reactor is adjusted by control valve VC 6 .

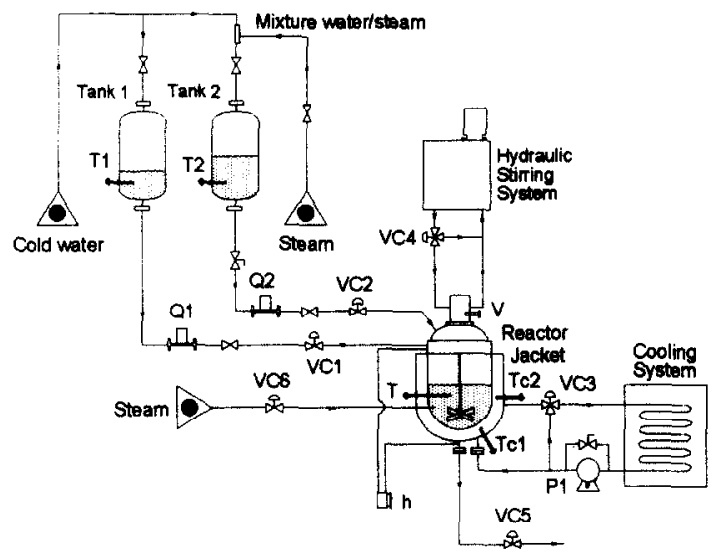

Figure 1 - Simplified flowsheet of the pilot plant

\section{Process Model}

The model equations can be written by deriving the appropriate mass and energy balances. Assuming constant density, the total mass balance leads to:

$$
\frac{\mathrm{dh}}{\mathrm{dt}}=\frac{1}{\mathrm{~A}}(\mathrm{Q} 1+\mathrm{Q} 2-\mathrm{Q} 3)
$$

The outflow Q3 is a function of both reactor level and VC5 position, and has been approximated by:

$$
\mathrm{Q} 3=\mathrm{C}_{\mathrm{V}} \sqrt{\mathrm{h}+\mathrm{h}_{\mathrm{o}}} \frac{\alpha \mathrm{VC5}}{1+\mathrm{VC5}(\alpha-1)}
$$

As mentioned before, although there is no exothermic chemical reaction taking place in the reactor, any desired zero order kinetics can be simulated, by injecting steam directly into the mixture. It has been experimentally verified (Afonso, 1997) that the power released can be approximated by:

$P_{\text {Stear }}=c \cdot \operatorname{Exp}(d \cdot V C 6)$

Assuming perfect mixing, the temperature inside the reactor can be described by the following energy balance:

$$
\begin{aligned}
& \frac{d T}{d t}=\frac{Q 2(T 2-T)}{A h}+\frac{Q 1(T 1-T)}{A h} \\
& -U_{g}(A+\pi d r b) \frac{(T-T c)}{\rho C_{p A h}}+\frac{\Delta H}{\rho C_{p}} k_{0} e^{-\frac{E_{a}}{R T}}
\end{aligned}
$$

The global heat transfer coefficient, $\mathrm{Ug}$, has been approximated by:

$$
\mathrm{U}_{\mathrm{g}}=\mathrm{a}_{1} \mathrm{VC} 3+\mathrm{a}_{2} \mathrm{Tc}+\mathrm{a}_{3} \mathrm{~T}+\mathrm{a}_{4}
$$

\section{Fault Detection and Identification Step}

During normal operation the process parameters can be tracked with some allowable tolerance due to process noise and model mismatch. The changes in these features are then determined with reference to the normal process, and subsequently used to recognise the fault and the time of its occurrence. In this case, this is accomplished by using a statistical decision test
(Afonso et al., 1995) based on a conventional hypothesis test.

For each one of the parameters involved, the hypothesis test is based on the change of their variance $\sigma^{2}$. In this case, the decision between the following two hy. potheses:

$$
\begin{array}{ll}
\mathrm{H}_{0}: \sigma_{1}^{2}=\sigma_{2}^{2} & \text { (no fault) } \\
\mathrm{H}_{1}: \sigma_{1}^{2} \neq \sigma_{2}^{2} & \text { (fault) }
\end{array}
$$

is determined by the ratio of the variances of the two samples

$$
\mathrm{F}_{0}=\frac{\mathrm{S}_{1}^{2}}{\mathrm{~S}_{2}^{2}}
$$

$\mathrm{H}_{0}$ is rejected if $\mathrm{F}_{0}$ violates the confidence levels defined by an $F$ distribution with $n_{1-1}$ and $n_{2}-1$ degrees of freedom, for a determined significance level, where $\mathrm{n}_{1}$ and $\mathrm{n}_{2}$ are the dimensions of the sample intervals.

This strategy is particularly suited for the detection of abrupt failures, as these have a major effect on the estimates of the process parameters. Typically, abrupt faults play an important role in safety-relevant systems, where hard-failures have to be detected early enough to avoid catastrophic results (Frank, 1990).

Therefore, in order to improve the reliability of the fault identification scheme, an EKF state estimator provides redundant values for the state variables. The fault identification is achieved by comparing the estimates provided by the EKF with the measurements obtained from the process plant. By this way it is possible to validate the fault occurrence and identify it. This procedure is only valid for the variables observed by the state estimator. For the remaining set of process variables the EKF must include additional states.

\section{Fault Tolerant Control Strategy}

Many sophisticated model based control algorithms have been developed under the assumption that all system components will not fail. Once a failure occurs, the system performance will degrade and may lead to divergence, generating dangerous situations, particularly when the failure occurs in the control loops.

Generally speaking, the fault tolerant control can be seen as a complex sequential procedure involving a few steps: fault detection, identification, and decision tasks. In the later, the control strategy is redefined in order to guarantee safe plant operation, or in other words, the control of the process is maintained under acceptable limits during the faulty period.

After the fault detection and the subsequent identification of the defective sensor, this is immediately disabled. The fault tolerant control is then achieved through an estimated measurement obtained by means of an EKF.

It is well known that methods based on state estimation using EKF or observers, rely on the use of accurate models, and on numerous assumptions such as known model structure and parameters, known noise characteristics and known effect of the faults on the model. In this work, one is faced with a challenging problem due to the fact that real data is involved. 


\section{Experimental Results}

To assess the proposed strategy in a practical environment, a set of experiments was carried out in the pilot plant. In all test runs the system was excited during the first 30 minutes by introducing disturbances in the level and temperature setpoints and in the flow rate $\mathrm{Q} 2$, in order to facilitate the initial process parameter identification. The physical parameters of the system and nominal operating conditions are given in Table 1.

Table 1-Physical parameters and operating conditions

\begin{tabular}{|lll|}
\hline $\mathrm{A}=0.166 \mathrm{~m}^{2}$ & $\mathrm{C}_{\mathrm{p}}=4148 \mathrm{~J} / \mathrm{KgK}$ & $\mathrm{h}_{0}=1.0 \mathrm{~m}$ \\
$\mathrm{a}_{1}=-398.8$ & $\mathrm{C}_{\mathrm{v}}=9.32 \cdot 10^{-5}$ & $\mathrm{k}_{0}=6.20 \cdot 10^{14} \mathrm{~mol} / \mathrm{m}^{3} \mathrm{~s}$ \\
$\mathrm{a}_{2}=107.8$ & $\mathrm{~d}=3.22$ & $\Delta \mathrm{H}_{\mathrm{g}}=-33488 \mathrm{~J} / \mathrm{mol}$ \\
$\mathrm{a}_{3}=-102.8$ & $\mathrm{~d}=0.46 \mathrm{~m}$ & $\alpha=13.7$ \\
$a_{4}=1821$ & $\mathrm{E}_{\mathrm{v}} / \mathrm{R}=-10000^{\circ} \mathrm{K}$ & $\rho=1000 \mathrm{Kg} / \mathrm{m}^{3}$ \\
$\mathrm{c}=1203$ & & \\
\hline
\end{tabular}

The fault detection method adopted here is based on the statistical properties of the-most sensitive parameters of the process model, using just one parameter for each model equation. After evaluating the sensitivity profiles of level and temperature to physical process parameters ( $\mathrm{pl}, \ldots, \mathrm{p} 4)$ defined according to eq.(9), the choice led to $\mathrm{p} 2$ and $\mathrm{p} 4$ (Afonso et al., 1995).

$\mathrm{p} 1=\frac{1}{\mathrm{~A}}, \mathrm{p}^{2}=\mathrm{C}_{\mathrm{v}}, \mathrm{p}^{3}=\alpha$, and $\mathrm{p} 4=\frac{\mathrm{U}_{\mathrm{g}}}{\rho \mathrm{C}_{\mathrm{p}}}$

The first two experiments with faults are illustrated in Figs. 2 and 3, which correspond to upsets of different magnitudes deliberately introduced in the level and temperature sensors. Both tests lasted for 7200 s and the faults were introduced 3600 s after the beginning of the experiment. In Figs.2a) and 3a), h(real) and $T$ (real) are the true values of these process variables, while $h(E K F)$ and $T(E K F)$ are the corresponding EKF estimates. The remaining process variables are shown in Figs.2b), and Figs.3b) and c).

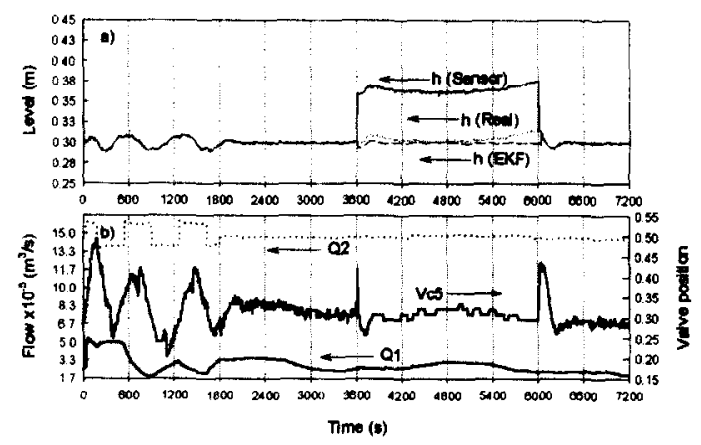

Figure 2 - Evolution of process variables after a fault in the level sensor.

The effects of a fault with an amplitude of $20 \%$ and a 40 minute span can be directly observed in the profile of $h$ (Sensor) (Fig.2a) or in the action of the level controller (see VC5 in Fig.2b). Here, the controller is initially misled by the wrong information from the sensor, but when the FDI mechanisms come successfully into action, the controller is fed by the EKF level estimate and is then able to sustain the system under control. As can be seen, the detection is very quick and the initial response of controller (induced by the fault) reveals how disastrous the results would have been if the FDI scheme did not perform appropriately. This effective behaviour of the detection mechanism is connected to the good performance of the parameter estimation system (in particular of $\mathrm{p} 2$ ), shown in Fig.4a).

Figure $4 \mathrm{~b}$ ) shows the results of the F-test for parameters $\mathrm{p} 2$ and $\mathrm{p} 4$. The horizontal lines correspond to the $99 \%$ confidence intervals for a set of 101 sequential samples (Box and Tiao, 1973). At $t=3600$ s the level sensor fault violates the confidence levels generating a fault alarm and therefore a detection. From this time on, by comparing $h$ (Sensor) with $h(E K F)$, one is able to identify the faulty sensor. This is thereafter disabled and its value replaced by the corresponding EKF estimate. The performance of the control system under the fault is highlighted in the interval between $t=3600$ s and $t=6000$ s of Figs 2 and can be regarded as quite satisfactory. This is because the controller is able to maintain the system under good control despite the erroneous information provided by the sensor.

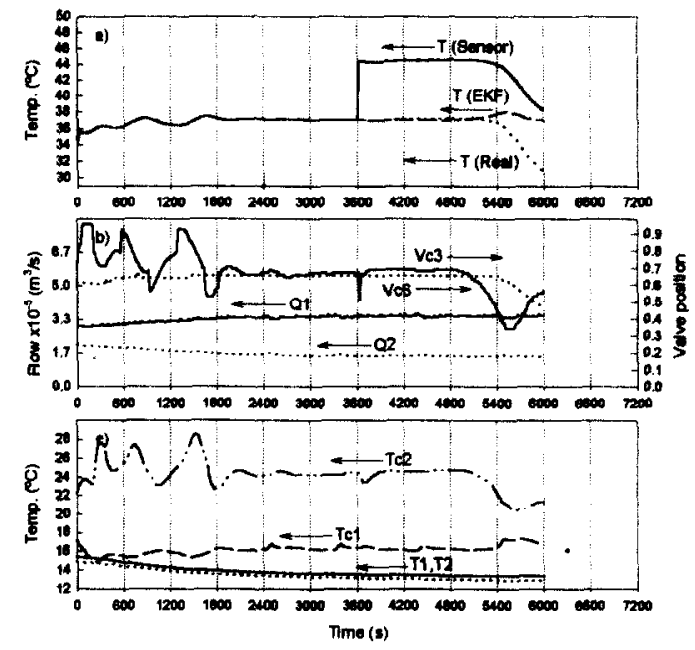

Figure 3 - Evolution of process variables after a fault in the reactor temperature sensor

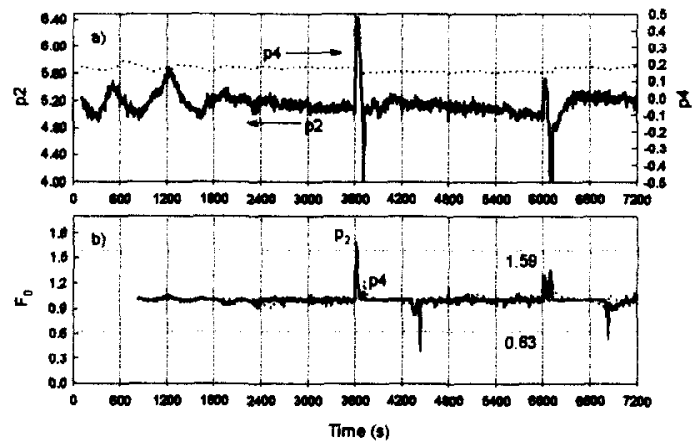

Figure $4-a)$ Estimates of parameter $p 2$ and $p 4$ corresponding to level fault. b) Statistical test corresponding to parameters $\mathrm{p} 2$ and $\mathrm{p} 4$.

Figure 3 illustrates the behaviour of the system when a failure is introduced into the temperature sensor of the main control loop at $t=3600 \mathrm{~s}$. The detection and identification mechanisms quickly play their role with success and control is carried on with the help of the 
EKF estimate. However this begins to diverge, approximately 1600 s after such event. The control system then becomes unable of keeping the process stable. Several other tests performed in the plant confirmed the insufficient model prediction capability, when faults occur in the temperature measurement. With the purpose of investigating the role played by the prevision model, a new test was performed whose results are shown in Fig.5. The following strategy was adopted: after the fault is detected, the process model used to predict the observations continues to be updated by the parameter estimator, contrarily to what happened in the previous set of experiments. This procedure is only possible because the correct measurements from the plant are available, as the faults are artificial generated. This is, however unfeasible in a situation of real failure.
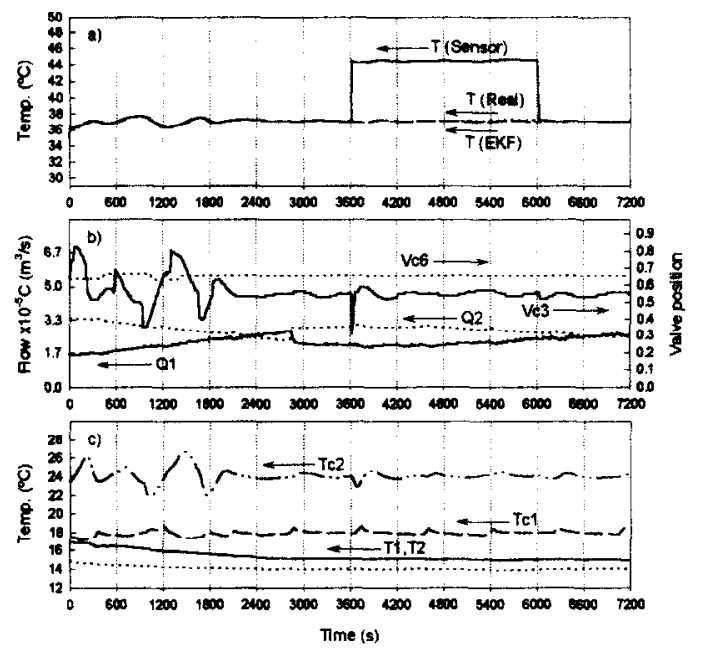

Figure 5 - Evolution of process variables after a fault in the temperature sensor when the adaptive mechanism is not switched off

From the analysis of Fig. 5 , the curves corresponding to $T(E K F)$ and $T$ (Real) are almost coincident, enabling the control system to effectively control the process for a much longer time. These results also mean that the modelling difficulties are not easily solved in the case of simulation a chemical reaction, which is believed to be the first cause of model mismatch in this particular example. In practice, the quantity of energy that is introduced into the reactor by steam injection is subjected to numerous and strong inaccuracies. The parameter estimator could compensate for this deviation in the steady state regime, but the variations in the steam flow during the fault period unbalance the model and are then responsible for the poorer quality of the estimates. In this last test, the adaptive mechanism is not switched off and therefore there is always a compensation of the model. This explains the good results obtained and simultaneously identifies the source of the problems mentioned above.

\section{Conclusions}

Several real control runs have been performed on a pilot plant processing a partially simulated exothermic chemical reaction. This methodology has the benefit of being readily adapted to experimentally test several control strategies, due to the low costs involved.

The FDI strategy highlighted here can validate the information that flows through instrumentation and consequently improves plant reliability.

The results obtained are encouraging, taking into account that the process is non-linear and that the plant can operate satisfactorily with a malfunctioning sensor. When the system becomes more complex and highly non-linear (as in the case of chemical reaction) leading to increased model uncertainties, the detection and identification mechanisms still exhibit a very effective performance. Such inaccuracies, however, give rise to poorer EKF estimates and therefore to shorter periods of stable operation without information from the sensor in the main control loop.

The good experimental results obtained, which exhibit simplicity, numerical robustness, and little computer effort, highlight their promising capabilities for industrial application. Additional work should be done, considering the fault identification related to sensors and actuators in the control loops, which will demand different control strategies.

\section{Acknowledgements}

Financial support from FLAD (Fundação LusoAmericana para o Desenvolvimento) and NARES (Natural Resins, Ltd.) is gratefully acknowledged. One of the authors is also thankful to JNICT (Junta Nacional de Investigação Científica e Tecnológica) through grant $\mathrm{BD} / 612 / 90-\mathrm{IB}$

\section{References}

Afonso, P.A., PhD Dissertation, University of Coimbra, (1997).

Afonso, P.A., J.L. Ferreira, J.A. Castro, and N.M. Oliveira, Proc. Dycord $95-4^{\text {th }}$ IFAC Symposium, 147152, Copenhagen, Denmark, (1995).

Afonso, P.A., N.M.C. Oliveira, and J.A.M. Castro, Comp. Chem. Eng., S769-S774, (1996).

Box, G. P., and G. C. Tiao, Addison-Wesley, (1973).

Frank, P.M., Automatica, 26, 459-474, (1990).

Gertler, J.J., IEEE Control Systems Magazine, 3-11, (1988)

Isermann, R., Automatica, 20, 387-404, (1984).

Kershenbaum, L.S, and Kittisupakorn P., Trans. IChemE, 72-A, 55-63, Jan (1994).

Stewart, W.E., M. Caracotsios, and J.P. Sorensen. AIChE J., 38(4), 641-650, (1992).

\section{Notation}

A - Reactor cross-sectional area

$a_{1}, a_{2}, a_{3}, a_{4}, c, d, \alpha$ - Process parameter

$\mathrm{C}_{\mathrm{p}}$ - Fluid heat capacity

$\mathrm{C}_{\mathrm{V}}$ - Process parameter

$\mathrm{dr}$ - Reactor diameter

$E_{a}-$ Activation energy

$\mathrm{h}$ - Height of liquid in the reactor

$h_{0}$ - Level offset

$k_{0}-$ Frequency factor

$\mathrm{R}$ - Gas constant

$\mathrm{T}$ - Fluid temperature in the reactor

$\mathrm{Tc}$ - Average coolant temperature

$\Delta t$ - Sampling time

$\rho$ - Fluid density 